\title{
Analysis of Single-Buffer Polling Models for Time-Slotted Communication Protocols
}

\author{
Hideaki Takagi \\ Institute of Socio-Economic Planning, University of Tsukuba \\ 1-1-1 Tennoudai, Tsukuba-shi, Ibaraki 305, Japan \\ phone: 81-298-53-5003; fax: 81-298-55-3849 \\ e-mail: takagi@shako.sk.tsukuba.ac.jp
}

\begin{abstract}
The message waiting time in a system of multiple queues, each with a single buffer, attended by a server in cyclic order is studied under the assumption that the time axis is slotted. The results can be used for the performance comparison with other slotted communication protocols. The distribution and the mean of the waiting time are obtained for general asymmetric systems, and closed-form expressions are derived for symmetric systems with constant service and switchover times. The simple results for the continuous polling model are identified with the limiting forms for infinitely many queues in stable unlimited-buffer systems with exhaustive, gated, or limited service discipline.
\end{abstract}

\section{Keywords}

Performance, local area networks, polling systems, queues.

\section{INTRODUCTION}

Polling models generally refer to systems of multiple queues served by nondedicated servers with rules that allocate the server to the queues. In particular, a basic polling model is a system of multiple queues attended by a single server in cyclic order. While the term polling originates in the polling data link control scheme in communication networks, generic models have been applied to the performance evaluation of other communication protocols such as token ring as well as to the performance modeling in the fields of computer operating systems, manufacturing, and transportation. Those readers who 
are interested in analysis and applications of polling models are referred to my earlier monograph [Takagi 1986] and surveys [Takagi 1988, 1990, 1991, 1994].

A system of multiple queues in which each queue has a buffer that can accommodate at most one message at a time is called a single-buffer model. Those messages that arrive to find the buffer occupied are lost. This model has been used for the performance modeling of various medium access protocols, including ALOHA [Lam and Kleinrock 1975], CSMA [Tobagi and Kleinrock 1977], CSMA/CD [Tobagi and Hunt 1980], and slotted ring [Jung and Un 1992; Yamazaki et al. 1993]. There are a number of studies for the single-buffer polling model, such as Mack et al. [1957], Scholl and Potier [1978], Takine et al. [1988], Ibe and Cheng [1989] only to mention a few representative ones. However, in all available studies of single-buffer polling models, the time axis is assumed to be continuous, in contrast with the assumption that the time is slotted which is adopted in the models of other communication protocols mentioned above. It is therefore interesting to investigate the slotted (or discrete-time) single-buffer polling model. The results can then be used for the performance comparison with other protocol on the same basis.

Specifically, the model analyzed in this paper is a system of $N$ queues each with a single buffer served by a server in cyclic order. The time axis of the system is segmented into a sequence of equal-size intervals of unit duration called slots. Service and switchover of the server can be started and ended only at slot boundaries so that their durations are always integral multiples of a slot. At an empty queue, say queue $i$, a message arrives with probability $\lambda_{i}$, or does not arrive with probability $1-\lambda_{i}$ in each slot, independently of all other events occurring in the system $\left(0<\lambda_{i}<1\right)$. Therefore, the mean interarrival time is given by $1 / \lambda_{i}$ slots. Let $X_{i}$ be the service time (measured in slots) at queue $i$. The probability generating function (PGF) $B_{i}(z)$ and the mean $b_{i}$ for $X_{i}$ are given by

$$
B_{i}(z):=\sum_{l=1}^{\infty} P\left\{X_{i}=l\right\} z^{l} \quad \text { and } \quad b_{i}:=E\left[X_{i}\right]=B_{i}^{(1)}(1)
$$

Similarly, let $R_{i}$ be the time (measured in slots) needed by the server to switch from queue $i$ to queue $i+1$ ( $R_{N}$ for the switch from queue $N$ to queue 1). The PGF $R_{i}(z)$ and the mean $r_{i}$ for $R_{i}$ are given by

$$
R_{i}(z):=\sum_{l=1}^{\infty} P\left\{R_{i}=l\right\} z^{l} \quad \text { and } \quad r_{i}:=E\left[R_{i}\right]=R_{i}^{(1)}(1)
$$

The mean of the total switchover time is given by

$$
R:=\sum_{i=1}^{N} r_{i}
$$

Our primary objective of analysis is to obtain the PGF $W_{i}(z)$ and the mean $E\left[W_{i}\right]$ for the message waiting time $W_{i}$ (measured in slots) at queue $i$ for $1 \leq i \leq N$.

This rest of this paper is organized as follows. In Section 2, we show an analysis to give $W_{i}(z)$ and $E\left[W_{i}\right]$ for $1 \leq i \leq N$. In principle, they can be obtained through the solution to a set of $N\left(2^{N-1}-1\right)$ linear equations. Explicit results are given when $N=2$. In Section 3, we consider a special case in which the switchover times are constant, the 
service times are constant and identical for all queues, and the arrival rates are also identical for all queues. Closed-form expressions are derived for $W(z)$ and $E[W]$. In Section 4 , we address the continuous polling model which can be obtained by considering the limit $N \rightarrow \infty$ while keeping the total arrival rate fixed in the model of Section 3 . Simple expressions for $E[W]$ and $\operatorname{Var}[W]$ are derived, and it is noted that they are the limiting forms for the corresponding results in stable infinite-capacity polling systems as $N \rightarrow \infty$.

\section{ASYMMETRIC SYSTEMS}

Let us derive several relationships for the performance measures in an asymmetric system. Focusing on queue $i$, we first note that the throughput $\gamma_{i}$, that is, the mean number of messages served per slot is related to the mean waiting time $E\left[W_{i}\right]$ by

$$
\gamma_{i}=\frac{1}{E\left[W_{i}\right]+b_{i}+1 / \lambda_{i}}
$$

This follows from an observation that the state of queue $i$ repeats renewal-type cycles of an empty period of mean duration $1 / \lambda_{i}$ and an occupied period with mean duration $E\left[W_{i}\right]+b_{i}$. If $\alpha_{i}$ denotes the probability that a message is found in queue $i$ when the server arrives there, the definition of the throughput yields

$$
\gamma_{i}=\frac{\alpha_{i}}{E[C]}
$$

where $E[C]$ is the mean polling cycle time. In turn, the mean polling cycle time and the mean intervisit time $E\left[V_{i}\right]$ of the server for queue $i$ are given by

$$
\begin{gathered}
E[C]=R+\sum_{k=1}^{N} \alpha_{k} b_{k} \\
E\left[V_{i}\right]=R+\sum_{k \neq i} \alpha_{k} b_{k}=E[C]-\alpha_{i} b_{i}
\end{gathered}
$$

From (2.1)-(2.4), we get the relation

$$
E\left[W_{i}\right]=\frac{E\left[V_{i}\right]}{\alpha_{i}}-\frac{1}{\lambda_{i}}
$$

Let $w_{i}(l)$ be the probability that the message waiting time at queue $i$ is $l$ slots, and let $v_{i}(l)$ be the probability that the server intervisit time for queue $i$ is $l$ slots, where $l=1,2, \ldots$. Their PGFs are defined by

$$
W_{i}(z):=\sum_{l=0}^{\infty} w_{i}(l) z^{l} \quad \text { and } \quad V_{i}(z):=\sum_{l=1}^{\infty} v_{i}(l) z^{l}
$$

Considering the slot during an intervisit time in which a message arrives, we get

$$
W_{i}(z)=\frac{\sum_{l=0}^{\infty} \lambda_{i}\left(1-\lambda_{i}\right)^{l} \sum_{m=1}^{\infty} v_{i}(l+m) z^{m-1}}{\sum_{l=0}^{\infty} \lambda_{i}\left(1-\lambda_{i}\right)^{l}\left[1-\sum_{m=1}^{l} v_{i}(m)\right]}
$$


which reduces to

$$
W_{i}(z)=\frac{\lambda_{i}\left[V_{i}(z)-V_{i}\left(1-\lambda_{i}\right)\right]}{\left[1-V_{i}\left(1-\lambda_{i}\right)\right]\left(z-1+\lambda_{i}\right)}
$$

We can get (2.5) from (2.8) by noting the relation

$$
\alpha_{i}=1-V_{i}\left(1-\lambda_{i}\right)
$$

The PGF $V_{i}(u)$ for the intervisit time can be obtained by considering the successive station times, where the station time $\omega_{k}$ for the $k$ th visited queue is defined as the sum of the switchover time from queue $k-1$ to queue $k$ and the possible service time at queue $k$. The joint distribution for $N$ successive station times is defined by

$$
\Omega_{k}\left(z_{1}, z_{2}, \ldots, z_{N}\right):=E\left[z_{1}^{\omega_{k-N+1}} z_{2}^{\omega_{k-N+2}} \cdots z_{N}^{\omega_{k}}\right]
$$

Since the intervisit time $V_{k}$ for the $k$ th visited queue is given by

$$
V_{k}=\omega_{k-N+1}+\omega_{k-N+2}+\cdots+\omega_{k-1}+R_{k-1}
$$

we get

$$
V_{k}(z)=\Omega_{k-1}(1, z, \ldots, z) R_{k-1}(z)
$$

From the state transition equations

$$
\begin{aligned}
& P\left\{\omega_{k-N+1}, \ldots, \omega_{k-1}, \omega_{k}\right\} \\
& =\sum_{\omega_{k-N}} P\left\{\omega_{k-N}, \omega_{k-N+1}, \ldots, \omega_{k-1}\right\}\left(1-\lambda_{k}\right)^{\omega_{k-N+1}+\cdots+\omega_{k-1}+\omega_{k}} P\left\{R_{k-1}=\omega_{k}\right\} \\
& +\sum_{\omega_{k-N}} P\left\{\omega_{k-N}, \omega_{k-N+1}, \ldots, \omega_{k-1}\right\}\left[1-\left(1-\lambda_{k}\right)^{\omega_{k-N+1}+\cdots+\omega_{k-1}+\omega_{k}-b_{k}}\right] \\
& \quad \times \sum_{b_{k}=1}^{\omega_{k}-1} P\left\{R_{k-1}=\omega_{k}-b_{k}\right\} P\left\{X_{k}=b_{k}\right\}
\end{aligned}
$$

we obtain

$$
\begin{aligned}
\Omega_{k}\left(z_{1}, \ldots, z_{N}\right) & =R_{k-1}\left[\left(1-\lambda_{k}\right) z_{N}\right]\left[1-B_{k}\left(z_{N}\right)\right] \Omega_{k-1}\left(1,\left(1-\lambda_{k}\right) z_{1}, \ldots,\left(1-\lambda_{k}\right) z_{N-1}\right) \\
& +R_{k-1}\left(z_{N}\right) B_{k}\left(z_{N}\right) \Omega_{k-1}\left(1, z_{1}, \ldots, z_{N-1}\right)
\end{aligned}
$$

From these equations, we can get a set of $N\left(2^{N-1}-1\right)$ equations for the same number of unknowns $\left\{\Omega_{k}\left(1,\left(1-\lambda_{k+1}\right)^{\nu_{1}}, \ldots, \Pi_{j=1}^{N-1}\left(1-\lambda_{k+j}\right)^{\nu_{j}}\right) ; \nu_{j}=0,1(1 \leq j \leq N-1), 1 \leq\right.$ $k \leq N\}$. Solving for them, we can calculate $\Omega_{k}(1, z, \ldots, z)$, which is used to obtain $V_{k}(z)$ and therefore $W_{k}(z)$.

As an example, consider the case in which $N=2$. Equations (2.14) then read

$$
\begin{aligned}
& \Omega_{1}\left(z_{1}, z_{2}\right)=R_{2}\left[\left(1-\lambda_{1}\right) z_{2}\right]\left[1-B_{1}\left(z_{2}\right)\right] \Omega_{2}\left(1,\left(1-\lambda_{1}\right) z_{1}\right)+R_{2}\left(z_{2}\right) B_{1}\left(z_{2}\right) \Omega_{2}\left(1, z_{1}\right) \\
& \Omega_{2}\left(z_{1}, z_{2}\right)=R_{1}\left[\left(1-\lambda_{2}\right) z_{2}\right]\left[1-B_{2}\left(z_{2}\right)\right] \Omega_{1}\left(1,\left(1-\lambda_{2}\right) z_{1}\right)+R_{1}\left(z_{2}\right) B_{2}\left(z_{2}\right) \Omega_{1}\left(1, z_{1}\right)
\end{aligned}
$$


which yield

$$
\begin{aligned}
& \Omega_{1}\left(1,1-\lambda_{2}\right)=\frac{R_{2}\left(1-\lambda_{2}\right) B_{1}\left(1-\lambda_{2}\right)+R_{1}\left(1-\lambda_{1}\right) R_{2}(\bar{\Lambda})\left[1-B_{1}\left(1-\lambda_{2}\right)\right] B_{2}\left(1-\lambda_{1}\right)}{1-R_{1}(\bar{\Lambda}) R_{2}(\bar{\Lambda})\left[1-B_{1}\left(1-\lambda_{2}\right)\right]\left[1-B_{2}\left(1-\lambda_{1}\right)\right]} \\
& \Omega_{2}\left(1,1-\lambda_{1}\right)=\frac{R_{1}\left(1-\lambda_{1}\right) B_{2}\left(1-\lambda_{1}\right)+R_{1}(\bar{\Lambda}) R_{2}\left(1-\lambda_{2}\right) B_{1}\left(1-\lambda_{2}\right)\left[1-B_{2}\left(1-\lambda_{1}\right)\right]}{1-R_{1}(\bar{\Lambda}) R_{2}(\bar{\Lambda})\left[1-B_{1}\left(1-\lambda_{2}\right)\right]\left[1-B_{2}\left(1-\lambda_{1}\right)\right]}
\end{aligned}
$$

where

$$
\bar{\Lambda}:=\left(1-\lambda_{1}\right)\left(1-\lambda_{2}\right)
$$

Since

$$
V_{1}(z)=\Omega_{2}(1, z) R_{2}(z) \quad \text { and } \quad V_{2}(z)=\Omega_{1}(1, z) R_{1}(z)
$$

it follows that

$$
\begin{aligned}
& \alpha_{1}=1-V_{1}\left(1-\lambda_{1}\right)=1-\Omega_{2}\left(1,1-\lambda_{1}\right) R_{2}\left(1-\lambda_{1}\right) \\
& \alpha_{2}=1-V_{2}\left(1-\lambda_{2}\right)=1-\Omega_{1}\left(1,1-\lambda_{2}\right) R_{1}\left(1-\lambda_{2}\right)
\end{aligned}
$$

and that

$$
\begin{aligned}
& E\left[V_{1}\right]=R+b_{2}\left[1-\Omega_{1}\left(1,1-\lambda_{2}\right) R_{1}\left(1-\lambda_{2}\right)\right]=R+\alpha_{2} b_{2} \\
& E\left[V_{2}\right]=R+b_{1}\left[1-\Omega_{2}\left(1,1-\lambda_{1}\right) R_{2}\left(1-\lambda_{1}\right)\right]=R+\alpha_{1} b_{1}
\end{aligned}
$$

We can use (2.20)-(2.24) in (2.5) and (2.8) to obtain the mean and the PGF for the waiting times $W_{1}$ and $W_{2}$. It is possible to derive similar expressions and conduct numerical computation for cases $N \geq 3$ as done by Ibe and Cheng [1989] for a continuous-time system.

\section{SYMMETRIC SYSTEMS WITH CONSTANT SERVICE AND SWITCHOVER TIMES}

We consider a special case in which all messages are of a fixed length $b$ and the switchover times from queue $i$ to queue $i+1$ are also constant $r_{i}$ for $1 \leq i \leq N$. Moreover, we assume that the arrival rates $\lambda$ of messages at all queues are the same.

For this system, let $\gamma$ be the total throughput of the system:

$$
\gamma=\sum_{i=1}^{N} \gamma_{i}
$$

From (2.1) and (2.2), we get

$$
\gamma=\frac{N}{E[W]+b+1 / \lambda}=\frac{E[Q]}{E[C]}
$$

where $E[Q]$ is the mean number of messages served in a polling cycle. Thus we have

$$
E[C]=R+b E[Q]
$$


From (3.2) and (3.3) we get

$$
E[W]=(N-1) b-\frac{1}{\lambda}+\frac{N R}{E[Q]}
$$

We can derive an explicit expression for $E[Q]$. To do so, we define the state $u_{k}$ of queue $k$ seen by the server as

$$
u_{k}:= \begin{cases}0 & \text { if queue } k \text { is empty } \\ 1 & \text { if queue } k \text { is occupied }\end{cases}
$$

Let us denote by $P_{i}\left(u_{1}, \ldots, u_{i-1}, u_{i}, u_{i+1}, \ldots, u_{N}\right)$ the probability that the server observes the states of queues $u_{i+1}, \ldots, u_{N}, u_{1}, \ldots, u_{i-1}$, and $u_{i}$ prior to arriving at queue $i$. The state transition equations are then given by

$$
\begin{aligned}
& P_{i}\left(u_{1}, \ldots, u_{i-1}, 1, u_{i+1}, \ldots, u_{N}\right) \\
& \quad=\left\{1-(1-\lambda)^{R+\left(u_{1}+\cdots+u_{i-1}+u_{i+1}+\cdots+u_{N}\right) b}\right\} \\
& \quad \times\left\{P_{i-1}\left(u_{1}, \ldots, u_{i-1}, 0, u_{i+1}, \ldots, u_{N}\right)+P_{i-1}\left(u_{1}, \ldots, u_{i-1}, 1, u_{i+1}, \ldots, u_{N}\right)\right\} \\
& P_{i}\left(u_{1}, \ldots, u_{i-1}, 0, u_{i+1}, \ldots, u_{N}\right) \\
& \quad=(1-\lambda)^{R+\left(u_{1}+\cdots+u_{i-1}+u_{i+1}+\cdots+u_{N}\right) b} \\
& \quad \times\left\{P_{i-1}\left(u_{1}, \ldots, u_{i-1}, 0, u_{i+1}, \ldots, u_{N}\right)+P_{i-1}\left(u_{1}, \ldots, u_{i-1}, 1, u_{i+1}, \ldots, u_{N}\right)\right\}
\end{aligned}
$$

By direct substitution, we can see that (3.6) and (3.7) are satisfied by

$$
P_{i}\left(u_{1}, \ldots, u_{N}\right)=K \prod_{j=0}^{\sum_{k=1}^{N} u_{k}-1}\left\{(1-\lambda)^{-(R+j b)}-1\right\} \quad \text { if } \sum_{k=1}^{N} u_{k}>0
$$

where

$$
K=P_{i}(0, \ldots, 0)
$$

is to be determined from the normalization condition.

From (3.8), we get

$$
P(n):=P\{Q=n\}= \begin{cases}K \\
K\left(\begin{array}{l}
N \\
n
\end{array}\right) \prod_{j=0}^{n-1}\left\{(1-\lambda)^{-(R+j b)}-1\right\} & \text { if } n=0 \\
\text { if } 1 \leq n \leq N\end{cases}
$$

Thus we determine

$$
K^{-1}=1+\sum_{n=1}^{N}\left(\begin{array}{l}
N \\
n
\end{array}\right) \prod_{j=0}^{n-1}\left\{(1-\lambda)^{-(R+j b)}-1\right\}
$$


Hence we obtain

$$
E[Q]=\frac{N \sum_{n=0}^{N-1}\left(\begin{array}{c}
N-1 \\
n
\end{array}\right) \prod_{j=0}^{n}\left\{(1-\lambda)^{-(R+j b)}-1\right\}}{1+\sum_{n=1}^{N}\left(\begin{array}{l}
N \\
n
\end{array}\right) \prod_{j=0}^{n-1}\left\{(1-\lambda)^{-(R+j b)}-1\right\}}
$$

Substituting (3.12) into (3.4), we get

$$
E[W]=(N-1) b-\frac{1}{\lambda}+\frac{R\left[1+\sum_{n=1}^{N}\left(\begin{array}{c}
N \\
n
\end{array}\right) \prod_{j=0}^{n-1}\left\{(1-\lambda)^{-(R+j b)}-1\right\}\right]}{\sum_{n=0}^{N-1}\left(\begin{array}{c}
N-1 \\
n
\end{array}\right) \prod_{j=0}^{n}\left\{(1-\lambda)^{-(R+j b)}-1\right\}}
$$

The PGF $V(z)$ for the intervisit time $V_{i}$ for queue $i$ can be derived as follows. Let $Q_{i}$ be the number of messages served during the intervisit time for queue $i$, which is given by

$$
Q_{i}=\sum_{k \neq i} u_{k}
$$

From (3.8) we get

$$
\begin{aligned}
& \sum_{u_{i}=0}^{1} P_{i}\left(u_{1}, \ldots, u_{N}\right) \\
& \quad= \begin{cases}K(1-\lambda)^{-R} & \text { if } \sum_{k \neq i} u_{k}=0 \\
K(1-\lambda)^{-\left(R+b \sum_{k \neq i} u_{k}\right)} \prod_{j=0}^{\sum_{k \neq i} u_{k}-1}\left\{(1-\lambda)^{R+j b)}-1\right\} & \text { if } \sum_{k \neq i} u_{k}>0\end{cases}
\end{aligned}
$$

Thus we obtain

$$
P\left\{Q_{i}=n\right\}= \begin{cases}K(1-\lambda)^{-R} & \text { if } n=0 \\
K\left(\begin{array}{c}
N-1 \\
n
\end{array}\right)(1-\lambda)^{-(R+n b)} \prod_{j=0}^{n-1}\left\{(1-\lambda)^{-(R+j b)}-1\right\} & \text { if } 1 \leq n \leq N-1\end{cases}
$$

Since

$$
V_{i}=R+Q_{i} b
$$

it follows that

$$
\begin{aligned}
V(z) & =E\left[z^{V_{i}}\right]=E\left[z^{R+Q_{i} b}\right]=z^{R} \sum_{n=0}^{N-1} z^{n b} P\left\{Q_{i}=n\right\} \\
& =K\left(\frac{z}{1-\lambda}\right)^{R}\left[1+\sum_{n=1}^{N-1}\left(\begin{array}{c}
N-1 \\
n
\end{array}\right)\left(\frac{z}{1-\lambda}\right)^{n b} \prod_{j=0}^{n-1}\left\{(1-\lambda)^{-(R+j b)}-1\right\}\right]
\end{aligned}
$$


Using (3.18), we can confirm that

$$
\begin{gathered}
\alpha=1-V(1-\lambda)=\frac{E[Q]}{N} \\
E[V]=R+(N-1) \alpha b
\end{gathered}
$$

As in (2.8), the PGF for the waiting time is given by

$$
W(z)=\frac{\lambda[V(z)-V(1-\lambda)]}{[1-V(1-\lambda)](z-1+\lambda)}
$$

which yields the mean waiting time $E[W]$ in (3.13).

\section{CONTINUOUS POLLING MODELS}

We can derive the expressions for the continuous polling model by taking the limit $N \rightarrow$ $\infty$ and $\lambda \rightarrow 0$ while keeping $N \lambda$ at a fixed value in the corresponding expressions for a symmetric system with constant service and switchover times [Coffman and Gilbert 1986].

Introducing

$$
\rho:=N \lambda b \quad \text { and } \quad \beta:=\frac{R}{b}
$$

we see that

$$
\left(\begin{array}{l}
N \\
n
\end{array}\right) \prod_{j=0}^{n-1}\left\{(1-\lambda)^{-(R+j b)}-1\right\} \rightarrow\left(\begin{array}{c}
-\beta \\
n
\end{array}\right)(-\rho)^{n} \text { as } N \rightarrow \infty
$$

Substituting this limit into (3.10) and (3.11), we get a negative binomial distribution

$$
P(n)=\left(\begin{array}{c}
-\beta \\
n
\end{array}\right)(-\rho)^{n}(1-\rho)^{\beta}, \quad n \geq 0
$$

for the number $Q$ of messages served in a polling cycle. The PGF for $Q$ is then given by

$$
Q(z):=\sum_{n=0}^{\infty} P(n) z^{n}=\left(\frac{1-\rho}{1-\rho z}\right)^{\beta}
$$

This yields the factorial moments

$$
\begin{gathered}
E[Q]=\frac{\rho \beta}{1-\rho}, \quad E[Q(Q-1)]=\frac{\rho^{2} \beta(1+\beta)}{(1-\rho)^{2}}, \\
E[Q(Q-1)(Q-2)]=\frac{\rho^{3} \beta(1+\beta)(2+\beta)}{(1-\rho)^{3}}
\end{gathered}
$$

The PGF $V(z)$ for the intervisit time, which is equivalent to the cycle time in the continuous model, is given by

$$
V(z)=z^{R} Q\left(z^{b}\right)
$$


which results in

$$
\begin{gathered}
E[V]=\frac{R}{1-\rho}, \quad E[V(V-1)]=\frac{R\{R-1+\rho(b+1)\}}{(1-\rho)^{2}}, \\
E[V(V-1)(V-2)]=\frac{R\left\{(R-1)(R-2)+\rho(b+1)(3 R+b-4)+\rho^{2}(b+1)(b+2)\right\}}{(1-\rho)^{3}}
\end{gathered}
$$

Taking the same limit in (3.21), we get

$$
W(z)=\frac{1-V(z)}{E[V](1-z)}
$$

Indeed, this is the PGF for the remaining intervisit time at a message arrival time. From (4.8) we have

$$
E[W]=\frac{E[V(V-1)]}{2 E[V]} \text { and } E[W(W-1)]=\frac{E[V(V-1)(V-2)]}{3 E[V]}
$$

Substituting (4.7) into (4.9), we obtain

$$
\begin{gathered}
E[W]=\frac{R+\rho b}{2(1-\rho)}-\frac{1}{2} \\
\operatorname{Var}[W]=\frac{(R+1)(R-1)+6 \rho(1+R b)+\rho(4+\rho)(b+1)(b-1)}{12(1-\rho)^{2}}
\end{gathered}
$$

We note that a symmetric polling system with infinite queueing capacities reduces to the continuous model in the limit $N \rightarrow \infty$, regardless of its service discipline. For example, the mean waiting times in stable symmetric polling systems with exhaustive, gated, and limited service disciplines are given by (see Takagi $[1985,1986]$ )

$$
\begin{gathered}
E[W]_{\text {exhaustive }}=\frac{R+\rho b-(\rho / N)(R+1)}{2(1-\rho)}-\frac{1}{2} \\
E[W]_{\text {gated }}=\frac{R+\rho b+(\rho / N)(R-1)}{2(1-\rho)}-\frac{1}{2} \\
E[W]_{\text {limited }}=\frac{R+\rho b+(\rho / N)(R-1-R / b)}{2[1-\rho-(\rho / N) R / b]}-\frac{1}{2}
\end{gathered}
$$

where we have assumed constant service and switchover times and Bernoulli arrivals. It is clear that the expressions in (4.12)-(4.14) approach the $E[W]$ in (4.10) as $N \rightarrow \infty$. On the other hand, closed-form expressions for the variance of the waiting time in polling systems with infinite queueing capacities are not available. However, for symmetric systems, they should approach $\operatorname{Var}[W]$ given in $(4.11)$ as $N \rightarrow \infty$. 


\section{CONCLUDING REMARKS}

The analysis in this paper consists of extensions of the existing techniques for continuoustime systems, namely, Mach et al. [1957] and Scholl and Potier [1978] for Section 2, Ibe and Cheng [1989] for Section 3, and Coffman and Gilbert [1986] for Section 4. However, these results only cannot be obtained by straightforward analogy with the corresponding results for continuous-time systems. In that sense, the results in this paper are new.

Several variations of the present model may be analyzed in a way similar to the treatment of continuous-time systems. They include Bernoulli feedback of messages, buffer relaxation model, and non-cyclic order of polling. See Takagi [1990] for references to these variations in continuous-time systems. Reduction in the number of equations to $2^{N-1}-1$ for an asymmetric system is also possible as done by Takine et al. [1988] for a continuous-time system.

\section{ACKNOWLEDGMENTS}

This work is supported in part by the Telecommunications Advancement Foundation. The author is grateful to the anonymous referees of the paper for many valuable comments.

\section{References}

[1] Coffman, E. G. Jr., and Gilbert, E. N. 1986. A continuous polling system with constant service times. IEEE Transactions on Information Theory, Vol.IT-33, No.4 (July), pp.584-591.

[2] Ibe, O. C., and Cheng, X. 1989. Performance analysis of asymmetric single-buffer polling systems. Performance Evaluation, Vol.10, No.1 (October), pp.1-14.

[3] Jung, W. Y., and Un, C. K. 1992. Analysis of throughput and delay of a high-speed slotted ring based on lumped modeling. IEEE Transactions on Communications, Vol.40, No.5 (May), pp.860-862.

[4] Lam, S. S., and Kleinrock, L. 1975. Packet switching in a multiaccess broadcast channels: dynamic control procedures. IEEE Transactions on Communications, Vol.COM-23, No.9 (September), pp.891-904.

[5] Mack, C., Murphy, T., and Webb, N. L. 1957. The efficiency of $N$ machines unidirectionally patrolled by one operative when walking time and repair times are constants. Journal of the Royal Statistical Society, Series B, Vol.19, No.1, pp.166172.

[6] Scholl, M., and Potier, D. 1978. Finite and infinite source models for communication systems under polling. IRIA Rapport de Recherche, No.308, Institut de Rescherche en Informatique et en Automatique, Le Chesnay, France. 
[7] Takagi, H. 1985. Mean message waiting times in symmetric multi-queue systems with cyclic service. Performance Evaluation, Vol.5, No.4 (November), pp.271-277.

[8] Takagi, H. 1986. Analysis of Polling Systems. The MIT Press, Cambridge, Massachusetts.

[9] Takagi, H. 1988. Queuing analysis of polling models. ACM Computing Surveys, Vol.20, No.1 (March), pp.5-28.

[10] Takagi, H. 1990. Queueing analysis of polling models: An update. In Stochastic Analysis of Computer and Communication Systems, H. Takagi (editor), pp.267318, Elsevier Science Publishers B. V. (North-Holland), Amsterdam.

[11] Takagi, H. 1991. Application of polling models to computer networks. Computer Networks and ISDN Systems, Vol.22, No.3 (October), pp.193-211.

[12] Takagi, H. 1994. Queueing analysis of polling models: progress in 1990-1993. In Frontiers in Queueing: Models, Methods and Problems, J. H. Dshalalow (editor), CRC Press. To appear.

[13] Takine, T., Takahashi, Y., and Hasegawa, T. 1988. Exact analysis of asymmetric polling system with single buffers. IEEE Transactions on Communications, Vol.36, No.10 (October), pp.1119-1127.

[14] Tobagi, F. A., and Hunt, V. B. 1980. Performance analysis of carrier sense multiple access with collision detection. Computer Networks, Vol.4, No.5 (OctoberNovember), pp.245-259.

[15] Tobagi, F. A., and Kleinrock, L. 1977. Packet switching in radio channels: part IV stability considerations and dynamic control in carrier sense multiple access. IEEE Transactions on Communications, Vol.COM-25, No.10 (October), pp.1103-1120.

[16] Yamazaki, G., Kobayashi, N., Takagi, H., and Wolff, R. W. 1993. Performance analysis of a slotted ring LAN by a single-buffer model. Transactions of the Institute of Electronics, Information and Communication Engineers, Vol.J76-B-I, No.4 (April), pp.340-348 (in Japanese).

\section{BIOGRAPHY}

Dr. Takagi is Professor at the Institute of Socio-Economic Planning of the University of Tsukuba, Japan. He received his B.S. and M.S. degrees both in Physics from the University of Tokyo in 1972 and 1974, respectively. He received his Ph.D. degree in Computer Science from the University of California, Los Angeles, in 1983. He was with IBM Japan from 1974 to 1993 . He is the author of research monographs Analysis of Polling Systems (The MIT Press, 1986), and Queueing Analysis : A Foundation of Performance Evaluation, volumes 1 through 3 (Elsevier, 1991, 1993 and 1993). He is a Senior Member of IEEE, and a Full Member of ORSA. He serves as editors for such journals as IEEE/ACM Transactions on Networking, Performance Evaluation, and Queueing Systems. 